\title{
Design and Clinical Verification of Surface Enhanced Raman Spectroscopy Diagnostic Technology for Individual Cancer Risk Prediction
}

Kevin M. Koo ${ }^{1 \ddagger}$, Jing Wang ${ }^{1 *}$, Renée S. Richards ${ }^{2,3}$, Aine Farrell ${ }^{2}$, John W. Yaxley ${ }^{2,4}$, Hema Samaratunga $^{2,5,6}$, Patrick E. Teloken ${ }^{4,6}$, Matthew J. Roberts ${ }^{2,4}$, Geoffrey D. Coughlin ${ }^{4}$, Martin F. Lavin ${ }^{2}$, Paul N. Mainwaring ${ }^{1}$, Yuling Wang ${ }^{7}$, Robert A. Gardiner ${ }^{2,4,8,9}$, and Matt Trau ${ }^{1,10^{*}}$

${ }^{1}$ Centre for Personalized Nanomedicine, Australian Institute for Bioengineering and Nanotechnology, University of Queensland, Brisbane, QLD4072, Australia

${ }^{2}$ The University of Queensland, Centre for Clinical Research, Brisbane, QLD4029, Australia

${ }^{3}$ QIMR Berghofer Medical Research Institute, Brisbane, QLD4006, Australia

${ }^{4}$ Department of Urology, Royal Brisbane and Women's Hospital, Brisbane, QLD4029, Australia

${ }^{5}$ Aquesta Specialized Uropathology, Brisbane, QLD4066, Australia

${ }^{6}$ Princess Alexandra Hospital, Brisbane, QLD4102, Australia

${ }^{7}$ Department of Molecular Sciences, Faculty of Science and Engineering, Macquarie University, Sydney, NSW2109, Australia

${ }^{8}$ Edith Cowan University, Perth, WA6027, Australia

${ }^{9}$ Griffith University, Brisbane, QLD4111, Australia

${ }^{10}$ School of Chemistry and Molecular Biosciences, University of Queensland, Brisbane, QLD4072, Australia

${ }^{\ddagger}$ These authors contributed equally to this work.

Corresponding Author: m.trau@uq.edu.au 


\section{ABSTRACT}

The use of emerging nanotechnologies, such as plasmonic nanoparticles in diagnostic applications, potentially offers opportunities to revolutionize disease management and patient healthcare. Despite worldwide research effort in this area, there is still a dearth of nanodiagnostics which have been successfully translated for real-world patient usage due to the predominant sole focus on assay analytical performance and lack of detailed investigations into clinical performance in human samples. In a bid to address this pressing need, we herein describe a comprehensive clinical verification of a prospective label-free surface-enhanced Raman scattering (SERS) nanodiagnostic assay for prostate cancer (PCa) risk stratification. This contribution depicts a roadmap of 1) designing a SERS assay for robust and accurate detection of clinically-validated PCa RNA targets; 2) employing a relevant and proven PCa clinical biomarker model to test our nanodiagnostic assay; 3 ) investigating the clinical performance on independent training $(n=80)$ and validation $(n=$ 40) cohorts of PCa human patient samples. By relating the detection outcomes to goldstandard patient biopsy findings, we established a PCa risk scoring system which exhibited a clinical sensitivity and specificity of 0.87 and 0.90 , respectively [area-under-curve of 0.84 (95\% confidence interval: 0.81-0.87) for differentiating high- and low-risk PCa] in the validation cohort. We envision that our SERS nanodiagnostic design and clinical verification approach may aid in the individualized prediction of PCa presence and risk stratification; and may overall serve as an archetypical strategy to encourage comprehensive clinical evaluation of nanodiagnostic innovations.

\section{KEYWORDS}

surface-enhanced Raman spectroscopy, silver nanoparticles, prostate cancer, nanotechnology, clinical, patient samples, MiPS 
The use of nanoparticles such as plasmonic surface-enhanced Raman scattering (SERS) nanoparticles or fluorescent quantum dots, has been shown to be promising for disease diagnostics applications. ${ }^{1-4}$ Yet, to date, there is still a dearth of nanoparticle-based diagnostics which have been translated for use in the clinic. This is partly attributed to the limited clinical technology verification studies for evaluating the clinical sensitivity and specificity (i.e. extent of false positives/negatives outcomes) of a diagnostic assay. 5,6 Typically, these clinical diagnostic metrics are obtained by testing on statistically-designed cohorts of annotated patient samples as a demonstration of clinical suitability. Progress on this front has been made by Chan and colleagues who recently reported a full clinical validation of QD barcode technology for diagnosing patients infected with Hepatitis B Virus (HBV). ${ }^{7}$ Ideally, this clinical validation concept could also be extended to the powerful SERS technique which has seen widespread use in biosensing research.

SERS has been demonstrated as a facile tool for direct nucleic acid (NA) biomarker analysis without the need for time-consuming, tedious, and costly extrinsic labeling. ${ }^{8-12}$ SERS involves the direct adsorption of NA sequences onto metallic nanostructured surfaces to generate selective sequence-dependent Raman spectral signatures. Due to recent advances in improving NA-SERS plasmonic substrate interactions, there have been vast improvements in SERS signal strength and reproducibility for NA biosensing. ${ }^{9,}{ }^{13}, 14$ This has led to a real possibility for translation of SERS technology for clinical use, as exemplified by an initial demonstration of SERS detection of RNA biomarkers in patient urine samples. ${ }^{15}$ However, it is still imperative to progress beyond these promising proof-of-concept analytical studies, and further investigate the clinical performance of SERS as a nanodiagnostic tool for patient use. To achieve this, SERS is to be ideally applied to a clinically-validated panel of disease biomarkers: a procedure which is rarely undertaken in the majority of nanodiagnostic publications. This has thus motivated us to undertake a comprehensive clinical verification of SERS by interrogating its clinical metrics with a well-suited cancer risk stratification scoring model.

Prostate cancer $(\mathrm{PCa})$ is the most commonly diagnosed male cancer, with early detection currently based on serum prostate specific antigen (PSA) to facilitate early treatment and improved survival. In spite of its widespread use, biological variation of the PSA biomarker in individuals has resulted in unreliable diagnostic accuracy, and associated side effects of unnecessary biopsies. ${ }^{16-19}$ In contrast, the Mi-Prostate Score (MiPS) is a PCa risk score test which was developed recently to improve the well-known deficiencies of PSA testing in early PCa detection. MiPS combines the urinary quantification of PCa-specific 
RNA targets, TMPRSS2:ERG (T2:ERG) and PCA3, to predict a patient's possibility of having PCa before biopsy diagnosis. Thus far, the combined detection of T2:ERG and PCA3 in the MiPS test has been clinically validated on around 2000 patients, and shown to provide a significantly better PCa risk assessment than serum PSA alone. ${ }^{20-24}$ Therefore, MiPS is an ideal NA biomarker-driven clinical model to implement SERS detection of T2:ERG and $P C A 3$ for early PCa risk prediction. Furthermore, MiPS is currently performed through lengthy and multi-step laboratory testing and has not been previously adopted by any sort of nanodiagnostic technique. Thus, we rationalized that MiPS clinical performance could benefit from the integration of a NA detection nanotechnology, such as ultrasensitive SERS, in terms of enhanced detection sensitivity and speed.

In this work, we described the use of a SERS nanodiagnostic technology for early PCa risk stratification by detecting T2:ERG and PCA3 PCa biomarkers in patient samples; and employed an appropriate MiPS clinical model to evaluate performance in a clinicallydriven manner. We first prepared cationic silver nanoparticles (AgNPs) to generate distinct and reproducible SERS signals from pre-amplified T2:ERG and PCA3. A comprehensive clinical assay verification was then performed on initial training $(n=80)$, and subsequent validation $(n=40)$ cohorts of well-annotated patient samples. By following the algorithm of the clinically-validated MiPS, the generated biomarker SERS intensities were subsequently used to develop a PCa risk score for head-to-head comparisons with gold-standard tissue biopsy outcomes. Using this strategy of evaluating SERS nanotechnology from a clinical perspective, we successfully i) tailored our SERS_MiPS nanodiagnostic assay based on a proven clinically-useful PCa risk scoring model; ii) showcased enhanced detection speed of MiPS through SERS integration; iii) demonstrated the translational feasibility of SERS for patient usage via clinical metrics.

\section{RESULTS AND DISCUSSION}

\section{Scheme}

The working scheme and clinical verification workflow of our PCa nanodiagnostic assay, which coupled SERS with clinically-validated MiPS biomarkers (termed as "SERS_MiPS"), is illustrated in Figure 1. Briefly, total RNA is extracted from patient urine samples, followed by parallel isothermal reverse transcription-recombinase polymerase amplification (RT-RPA) of three RNA targets (T2:ERG, PCA3, KLK2) (Figure 1a-i). This amplification step is employed to stabilize the native RNA targets in homogeneous double-stranded DNA (dsDNA) form, and to enhance resultant SERS signals. ${ }^{15}$ The purified dsDNA amplicons are 


\section{SERS Substrate Characterization and Optimization}

SERS is a surface spectroscopic technique for direct detection of adsorbed NA targets on a SERS substrate (typically metallic NPs) surface. The resulting signal strength of the adsorbed NA targets is dependent on multiple variables; including NP size and NA length. ${ }^{25,}{ }^{26}$ To achieve maximum SERS signals, we sought to optimize the size of cationic AgNPs for ultrasensitive target detection in patient samples.

We synthesized cationic AgNPs of different sizes, and compared their SERS signal enhancement for T2:ERG amplicons. Through adjusting the amount of reducing agents (NaBH4); we synthesized $20 \mathrm{~nm}, 40 \mathrm{~nm}$, and $60 \mathrm{~nm}$ AgNPs (Figure 2a-c). The particle size distributions (PSDs) were further characterized by differential centrifugal sedimentation which measured NP sizes based on particle weight. Consistent with TEM data, the major peak maxima of PSDs indicated AgNP sizes of $20 \mathrm{~nm}, 40 \mathrm{~nm}$, and $60 \mathrm{~nm}$ (Figure 2d). The corresponding surface plasmon resonance bands (Figure 2e) showed a red-shift with increasing AgNP size, $394 \mathrm{~nm}$ for $20 \mathrm{~nm}$ AgNPs, $400 \mathrm{~nm}$ for $40 \mathrm{~nm}$ AgNPs and $413 \mathrm{~nm}$ for $60 \mathrm{~nm}$ AgNPs). This set of characterization experiments authenticated the successful synthesis of AgNPs with well-controlled sizes.

As SERS enhancement is dependent on the SERS substrate size, we observed stronger SERS signals for adsorbed T2:ERG amplicons on $40 \mathrm{~nm}$ AgNPs than on $20 \mathrm{~nm}$ AgNPs. 
However, for adsorbed T2:ERG amplicons on $60 \mathrm{~nm}$ AgNPs, SERS signals diminished due to excess NP aggregation and precipitation out of solution (Figure 2f). Therefore, cationic AgNPs at $40 \mathrm{~nm}$ was selected as the optimal SERS substrate to generate maximum signals for RNA biomarker detection in patient samples.

\section{Analytical Specificity}

The specificity of our methodology in detecting T2:ERG, $P C A 3$, and $K L K 2$ was first evaluated by assessing the isothermal amplification end-products of each target. Following rigorous primer design and testing on PCa cell lines, we verified using gel electrophoresis that each target amplification process generated corresponding amplicons of an expected band size (Figure S1). This showed that each biomarker target could be specifically amplified from a heterogeneous mixture of cellular NA extract.

To demonstrate that SERS can specifically differentiate T2:ERG, PCA3, and KLK2 amplicon signals based on their selective molecular information, the SERS signals of T2:ERG, $P C A 3$, and KLK2 amplicons were subjected to chemometric analysis. We used principal component analysis (PCA) to reduce the dimensionality of a multidimensional data set while retaining characteristics of all spectra. As shown in Figure 2g, PCA successfully identified and clustered Raman signals of T2:ERG, $P C A 3$, and $K L K 2$ amplicons into three separate groups, thus displaying significant differences within their SERS signals $(\mathrm{P}<0.005)$.

\section{Analytical Sensitivity}

The limit-of-quantification (LOQ) for individual T2:ERG, $P C A 3$, and $K L K 2$ targets in patient samples was determined using serial dilutions ( 0 to 100000 copies) of each biomarker (Figure 3a-c). As 10 copies generated similar saturating signal levels to that of 100 copies, it was found that a LOQ of 100 synthetic RNA copies in a background of healthy patient urinary RNA extract was achieved for all biomarkers within a dynamic range of 100 to 10 000 copies (Figure 3d-f). With this LOQ, the combination of RT-RPA and SERS used in our methodology imparted an improvement in analytical speed as compared to the current MiPS test. The standard MiPS test is presently accomplished through the combination of an alternative isothermal amplification technique called transcription-mediated amplification (TMA), and a chemiluminescence readout. ${ }^{24}$ Although the LOQ of both our methodology and the current TMA-based test are comparable (Table S2), ${ }^{27}$ we demonstrate that our nanotechnology-based methodology has significantly streamlined the assay workflow (Fig. S2) and shortened the total assay time (90 min vs 3-4 hrs). This could be attributed to the 
superior speed and amplification efficiency of RT-RPA which,${ }^{28}$ as well as the simplistic and rapid detection of target sequences by SERS. Additionally, our methodology also showed good assay reproducibility with intra- and inter-assay variability of $9.5 \%$ and $11.0 \%$ respectively $(n=3)$.

\section{Disease Risk Scoring in Training Cohort}

After demonstrating the analytical performance of our SERS_MiPS methodology for T2:ERG, $P C A 3$, and $K L K 2$ detection, we proceeded to test its clinical performance on human samples. We firstly aimed to refine our SERS_MiPS risk scoring system for early PCa diagnosis and prognosis by establishing functional cut off SERS signal levels for accurate PCa risk prediction. For this purpose, we used a training cohort $(n=80)$ that consisted of urine samples from $60 \mathrm{PCa}$ patients and 20 healthy controls. Within the $60 \mathrm{PCa}$ patient samples, we further defined low- and high-risk PCa subgroups on the basis of the patients' biopsy outcomes. Using the standard pathological Gleason grading of the biopsy tissues, lowrisk PCa was defined as Gleason score (GS) $<7$ and high-risk PCa as GS $\geq 7$. As shown in Table 1, $36(60 \%)$ and $24(40 \%)$ of the PCa patients were in the low- and high-risk PCa subgroups, respectively.

As depicted in the heat map diagram (Figure 4a), we obtained varying raw SERS signal intensities for the detection of T2:ERG, PCA3, and $K L K 2$ in the training cohort of patient urine samples. Using the spectral peak at $742 \mathrm{~cm}^{-1}$ (assigned to purines), we quantified the raw SERS intensities as representative of the expression levels of each biomarker. The expression levels of T2:ERG (Figure 4b) and PCA3 (Figure 4c) were then normalized by the level of $K L K 2$ (to control for varying number of prostate cells and therefore, amount of prostate RNA amongst the patient samples). We also performed qPCR of the three biomarkers from the same patient samples as a separate validation tool (Table S2), and attained good concordance between the qPCR and SERS biomarker expression data via Passing and Bablok regression analysis (Figure S3). ${ }^{29}$ It is also worth noting that the SERS and qPCR measurements were performed by different operators to ensure unbiased validation.

In accordance with the well-established MiPS PCa risk scoring algorithm, ${ }^{24}$ the normalized SERS signals were then utilized to calculate a "\%SERS_MiPS" value to estimate the risk of disease in the patients (Figure 4d). By relating the \%SERS_MiPS to the patients' biopsy GS results (Table S3), we found that all the healthy controls exhibited low $\%$ SERS_MiPS, and higher \%SERS_MiPS was correlated with higher probability of a 
positive PCa biopsy. Patients in high-risk PCa subgroups were also found to have overall higher \%SERS_MiPS as compared to healthy controls and low-risk PCa subgroup patients (Figure 4e). Based on the \%SERS_MiPS segregation for different PCa risk groups (Figure 4e), we primarily established a cut off of $20 \%$ SERS_MiPS, at which above an individual is scored PCa-positive and below which a patient is considered PCa-negative (Figure 4e). We also further set a cut off of $80 \%$ SERS_MiPS for differentiating low- and high-risk PCa groups (Figure 4e).

To evaluate clinical performance in the training cohort, we further analyzed the data to define clinical sensitivity (ability to detect a true positive, i.e. patients with PCa) and specificity (ability to detect a true negative, i.e. patients without PCa). Using the established cut off limits, the clinical sensitivity and specificity were 0.91 and 0.95 respectively within the training cohort. To investigate the clinical utility of our SERS_MiPS methodology in estimating patient risk for developing low- or high-risk PCa, we also plotted the clinical sensitivity vs 1-clinical specificity (Figure 4f) to generate a Receiver Operating Characteristic (ROC) plot. On the ROC plot, the area-under-curve (AUC) is indicative of test performance, whereby a value closer to 1 represents an ideal perfect test. Using SERS-MiPS, we measured an AUC of 0.94 (95\% confidence interval (CI): 0.88-0.98) for the ability to discriminate between patients with GS $<7$ and $\mathrm{GS} \geq 7$.

\section{Clinical Metric Evaluation in Validation Cohort}

For a more in-depth validation of our developed SERS_MiPS, we used an independent validation cohort $(n=40)$ to verify the clinical efficacy of \%SERS_MiPS cut off values. In addition, to provide a more realistic clinical screening scenario, the assay operator is blinded to the cohort's disease status and biopsy results to remove possible screening bias. This allows for a blind independent comparison of SERS_MiPS results with the reference biopsy results among a series of patients suspected (but not known) to have PCa until biopsy confirmation. Based on the subsequent biopsy data, the validation cohort was made up of 30 PCa patients with $18(60 \%)$ and $12(40 \%)$ in the low- and high-risk groups respectively, in addition to 10 healthy controls. In a similar fashion as to the training cohort, raw SERS signal intensities for T2:ERG, $P C A 3$, and $K L K 2$ were measured (Figure 5a) with qPCR validation (Table S3), normalized to corresponding T2:ERG (Figure 5b) and PCA3 (Figure 5c) levels, and generated into \%SERS_MiPS scores (Figure 5d). Using the established \%SERS_MiPS cut offs from the training cohort, we separated the patients into different risk groups (Figure 5e). Through comparisons with patients' biopsy outcomes (Table S4), we achieved a clinical 
sensitivity and specificity of 0.87 and 0.90 respectively, and the AUC for predicting high-risk $\mathrm{PCa}$ is 0.84 (95\% CI: 0.81-0.87) (Figure 5f).

\section{Clinical Performance of SERS_MiPS}

By fusing the nanotechnological advantages of SERS and the validated clinical benefit of MiPS to create SERS_MiPS, our study goal was to validate the potential of this nanodiagnostic tool for clinical implementation by performing a comprehensive clinical verification (Figure 1). The clinical verification serves to demonstrate that the \%SERS_MiPS score correlated with the clinical outcome of interest; such as specific detection of PCapositive cases to reduce unnecessary biopsies and treatments, as well as accurate discrimination of high-risk PCa among the positive cases.

We used independent training and validation patient cohorts to perform a comprehensive clinical verification of SERS_MiPS. After first establishing the \%SERS_MiPS risk scoring system in the training cohort (Figure 4), the subsequent clinical metrics obtained from the blinded validation cohort is a better representation of true clinical performance. Our analysis showed that SERS_MiPS displayed very high clinical sensitivity (0.87) and specificity (0.90) for detecting positive PCa cases (Figure 5). The high clinical specificity is especially useful for avoiding false PCa-negative patients, and highlighted the excellent disease specificity of the biomarkers used in MiPS. By using a cut off of 80 \%SERS_MiPS, we also achieved discrimination between high- and low-risk PCa with an AUC of 0.82. This AUC is similar/potentially higher to the MiPS-related evaluations conducted by Leyten et al. $(\mathrm{AUC}=0.84)^{22}$, Tomlins et al. $(\mathrm{AUC}=0.79)^{24}$ and Cornu et al . $(\mathrm{AUC}=0.74) .{ }^{23}$ Although differences in patient cohorts and assay techniques limit direct comparisons, it is evident that the use of MiPS significantly improves traditional PSA-based PCa testing and remains as a viable clinical model for evaluating our SERS_MiPS nanodiagnostic assay.

Notably, the clinical metrics obtained in this study (along with the high analytical sensitivity, specificity, and speed imparted by SERS technique), have fulfilled the objective of this study in showcasing the potential of SERS_MiPS for clinical translation. Thus, this current investigation into the clinical diagnostic effect of SERS_MiPS may warrant the further follow-up of a larger population-level study with disease prevalence being taken into consideration.

Furthermore, the \%SERS_MiPS risk scoring system for PCa could be useful in classifying patients during screening and guiding treatment decisions. Given the slow- 
growing nature of most PCa tumors, it is conceivable that low \%SERS_MiPS scoring may play an invaluable role for monitoring patients managed by active surveillance, whilst high \%SERS_MiPS scoring could identify patients for definitive and urgent evaluation.

\section{CONCLUSIONS}

In conclusion, this work is a comprehensive clinical verification report of a SERS nanodiagnostic technology, SERS_MiPS, by use of a clinically-validated MiPS risk stratification scoring model biomarker model. Our study encompassed i) implementation of a SERS nanotechnology for a prominent PCa diagnosis issue, ii) innovative use of MiPS as a relevant clinical biomarker model, and iii) detailed study design with two independent cohorts for comprehensive clinical verification of SERS_MiPS. As the majority of promising nanodiagnostic technologies rarely progress beyond the academic research stage, this contribution represents an effort to breakthrough this barrier by demonstrating the clinical capability of SERS_MiPS for genuine patient usage. The clinical performance of SERS_MiPS has been robustly interrogated with both training and validation cohorts, and the generated clinical metrics demonstrated the potential of SERS_MiPS for improving risk stratification in PCa patients.

There are several avenues for building upon the outcomes of this contribution. From a clinical standpoint, it is imperative that the clinical studies be progressively up-scaled to evaluate SERS_MiPS at a greater population level. This ensuing step will also call for multiinstitutional studies to further cross-validate the nanodiagnostic technology. From a technological perspective, the emergence of handheld Raman spectrometers could enable SERS_MiPS to be increasingly user-friendly and amenable for point-of-care use. In addition, to exploit the multiplexing capability of SERS, our current proposed system could be further streamlined with greater analytical speed and convenience. This may be achieved by utilizing a multiplex target amplification process as well as developing advanced methods to decipher multiplex SERS spectra, but taking care to maintain appropriate analytical and clinical assay performance.

We anticipate that our careful study design, in incorporating a viable clinical risk stratification model (MiPS) and using two independent cohorts (training and validation) for a clinical verification of a nanodiagnotic technology, could serve as an archetypical strategy to encourage more studies towards clinical translation of promising innovations from nanotechnology laboratories for more widespread use. 


\section{MATERIALS AND METHODS}

\section{Materials}

All reagents were purchased from Sigma Aldrich, unless otherwise stated. Synthetic oligonucleotide and primer sequences used in our experiments were obtained from Integrated DNA Technologies (Singapore), and sequences are shown in Table S1.

\section{Cationic SERS Substrate Synthesis and Characterization}

Cationic AgNPs were prepared as previously reported with ice-cold solutions. Briefly, $20 \mu \mathrm{L}$ of $0.5 \mathrm{M} \mathrm{AgNO}_{3}$ was added to $10 \mathrm{~mL}$ of distilled water, followed by the addition of $7 \mu \mathrm{L}$ of $100 \mathrm{mM}$ spermine. The mixture was vigorously stirred for $1 \mathrm{~min}$. Then, various amounts of $10 \mathrm{mM} \mathrm{NaBH}_{4}$ were added into the mixture to synthesize AgNPs of different sizes.

TEM images were taken with a Hitachi HT7700 microscope (Hitachi, Japan) operated at $120 \mathrm{kV}$. The size distribution of AgNPs was measured by differential centrifugal sedimentation with a disc centrifuge (model DC24000 UHR) from CPS instrument Inc. The disc was loaded with $14.4 \mathrm{~mL}$ of sucrose gradient fluid comprising of 8-24 wt.\% sucrose in water. The average density, refractive index, and viscosity of the sucrose gradient fluid were $1.069 \mathrm{~g} / \mathrm{mL}, 1.36$, and $1.505 \mathrm{cP}$, respectively. A disc rotational frequency of $24000 \mathrm{rpm}$ was used for the measurement of AgNPs. The localized surface plasmon resonance of AgNPs was -Mediated

\section{Clinical Sample Collection and Preparation}

Ethics approval was obtained from The University of Queensland Institutional Human Research Ethics Committee (Approval No. 201400012), and informed consent was obtained from all subjects prior to sample collection. De-identified voided urinary samples were prospectively collected from male patients prior to PCa needle biopsies, and healthy young men with no PCa family history. The training cohort consisted of 60 urine samples from men referred for needle biopsy and 20 healthy controls. The validation cohort consisted of urine samples from 30 men presenting for needle biopsy and 10 healthy controls. The clinicopathological characteristics are summarized in Table 1.

Total RNA in the urinary samples was extracted using the commercially-available ZR urine RNA isolation $\mathrm{Kit}^{\mathrm{TM}}$ (Zymo Research, USA). Briefly, urinary cells were enriched by passing $30 \mathrm{~mL}$ of urine, followed by $700 \mu \mathrm{L}$ of supplied urine RNA buffer through the supplied ZRC GF ${ }^{\mathrm{TM}}$ Filter. Collected urinary cells were then lyzed, washed and eluted in 10 $\mu \mathrm{L}$ of RNase-free water according to manufacturer's instructions. 


\section{Isothermal Amplification of MiPS Biomarkers}

The TwistAmp Basic RT kit (Twist-DX, UK) was used with slight modifications to manufacturer's instructions to specifically amplify each target RNA biomarker. Briefly, $1 \mu 1$ of urinary extracted total RNA and $250 \mathrm{nM}$ of target-specific (T2:ERG, PCA3, or KLK2) primers (Table 1) were added to the supplied reagents to make a $12.5 \mu \mathrm{l}$ of reaction volume. Each reaction was then incubated at $41^{\circ} \mathrm{C}$ for $15 \mathrm{~min}$. Afterwhich, amplicons were purified using the Agencourt AMPure XP SPRI kit (Beckman Coulter, USA), and eluted in RNasefree water for SERS measurements or amplicon size validation by agarose gel electrophoresis.

\section{SERS Measurements}

$1 \mu \mathrm{L}$ of target amplicon was incubated with $60 \mu \mathrm{l}$ of cationic AgNPs for 15 min prior to SERS measurements. SERS measurements were performed using a portable IM-52 Raman Microscope with a $70 \mathrm{~mW}, 785 \mathrm{~nm}$ laser for excitation. The SERS spectra were collected in solution at $1 \mathrm{~s}$ illumination to minimize signal variations, and in the range of $400-1800 \mathrm{~cm}^{-1}$. Each sample was represented with the average spectrum of 10 measurements in the whole sampled volume.

\section{\%SERS_MiPS Score Calculation}

For each sample, T2:ERG and $P C A 3$ SERS signals were normalized to KLK2 SERS signal, in order to correct for the number of prostate cells in a single sample. For example:

Normalized T2:ERG SERS signal = T2:ERG SERS signal $/$ KLK2 SERS signal

Based partially on the algorithm of the standard clinical MiPS test, ${ }^{24}$ we calculated our \%SERS_MiPS Score through association with pathological data from the training cohort:

$100 \% *\{[2 *$ (normalized urine T2:ERG SERS signal) + (normalized urine PCA3 SERS signal)] / 3 \}

\section{Chemometric Analysis}

The raw SERS spectra were firstly baseline-corrected to remove background noise using the Vancouver Raman Algorithm (a five-order polynomial fitting algorithm). ${ }^{30}$ As each SERS 
spectrum in the range of $400-1800 \mathrm{~cm}^{-1}$ possessed 1401 variables, PCA was then applied to capture main variables that were represented by principal components (PCs). Spectrum $i$ is in the form as:

$$
S_{i}=\sum_{j} s_{i j} * P C_{j}+E
$$

where $S_{i}$ represents spectrum $i$ and is a combination of parameters, $s_{i j}$ values are PC scores and $E$ is the residual. The data $P C_{j}$ is highly correlated in a multi-dimensional space.

PC1 and PC2 accounted for $>80 \%$ of total spectral characteristics in the entire data set of T2:ERG, $P C A 3$, and $K L K 2$ amplicon, and were thus selected to represent their SERS spectral characteristics. SPSS 19.0 software package (SPSS Inc., Chicago, Illinois) was used for PCA.

\section{qPCR Validation}

The KAPA SYBR ${ }^{\circledR}$ FAST One-Step qRT-PCR kit (KAPA Biosystems, Australia) was used to set up a single reaction volume of $10 \mu 1$ for each sample. Each reaction volume consists of 1X KAPA SYBR ${ }^{\circledR}$ FAST qPCR Master Mix, $200 \mathrm{nM}$ of each forward and reverse primer (Table 1), 1X KAPA RT Mix, $50 \mathrm{nM}$ ROX dye and $30 \mathrm{ng}$ of cell line total RNA template. RT-qPCR was performed using the Applied Biosystems ${ }^{\circledR} 7500$ Real-Time PCR System (Thermo Fisher Scientific, Australia). The cycling protocol was: $42^{\circ} \mathrm{C}$ for $10 \mathrm{~min}$ to synthesize cDNA, followed by $95^{\circ} \mathrm{C}$ for $5 \mathrm{~min}$ to deactivate RT before cycling 35 times $\left(95^{\circ} \mathrm{C}\right.$ for $30 \mathrm{~s}, 50^{\circ} \mathrm{C}$ for $30 \mathrm{~s}$ and $72^{\circ} \mathrm{C}$ for $1 \mathrm{~min}$ ) and finished with $72^{\circ} \mathrm{C}$ for $10 \mathrm{~min}$.

\section{ACKNOWLEDGEMENTS}

The authors acknowledge grants received by our laboratory from the National Breast Cancer foundation of Australia (CG-12-07), ARC DP (140104006), ARC DP (160102836) and 2018 Royal Brisbane Women's Hospital Foundation Research Project Grant. These grants have significantly contributed to the environment to stimulate the research described here. K.M.K. and J.W acknowledge support from the Australian Government Research Training Program Scholarships. 
Table 1 Clinical characteristic summary of patients in training and validation cohorts. $I Q R$ interquartile range, $P S A$ prostate specific antigen, GS Gleason score, $A U C$ area-undercurve, $C I$ confidence interval.

\begin{tabular}{|c|c|c|c|c|}
\hline & \multicolumn{2}{|c|}{ Training Cohort $(n=80)$} & \multicolumn{2}{|c|}{ Validation Cohort $(n=40)$} \\
\hline & $\begin{array}{l}\text { Healthy } \\
\text { Controls }\end{array}$ & $\begin{array}{l}\text { Prostate } \\
\text { Cancer }\end{array}$ & $\begin{array}{l}\text { Healthy } \\
\text { Controls }\end{array}$ & $\begin{array}{l}\text { Prostate } \\
\text { Cancer }\end{array}$ \\
\hline Samples $(n)$ & $20(25 \%)$ & $60(75 \%)$ & $10(25 \%)$ & $30(75 \%)$ \\
\hline Age (years) (mean (median:IQR)) & $\begin{array}{c}35(31: 27- \\
50)\end{array}$ & $61(62: 56-72)$ & $\begin{array}{c}32(33: 28- \\
46)\end{array}$ & $\begin{array}{c}62.5(61: 29- \\
74)\end{array}$ \\
\hline Median PSA (ng/mL) (IQR) & $2.8(0.5-5.6)$ & $8.9(3.0-13.9)$ & $\begin{array}{c}2.6(0.4- \\
5.9)\end{array}$ & $10.1(2.0-12.3)$ \\
\hline \multicolumn{5}{|l|}{ Gleason Score } \\
\hline $\mathbf{G S}<7$ & NA & $36(60 \%)$ & NA & $18(60 \%)$ \\
\hline GS $\geq 7$ & NA & $24(40 \%)$ & NA & $12(40 \%)$ \\
\hline Clinical Sensitivity & \multicolumn{2}{|c|}{0.91} & \multicolumn{2}{|c|}{0.87} \\
\hline Clinical Specificity & \multicolumn{2}{|c|}{0.95} & \multicolumn{2}{|c|}{0.90} \\
\hline AUC for High-Risk PCa Detection & \multicolumn{2}{|c|}{0.94 (95\% CI: 0.88-0.98) } & \multicolumn{2}{|c|}{0.84 (95\% CI: 0.81-0.87) } \\
\hline
\end{tabular}




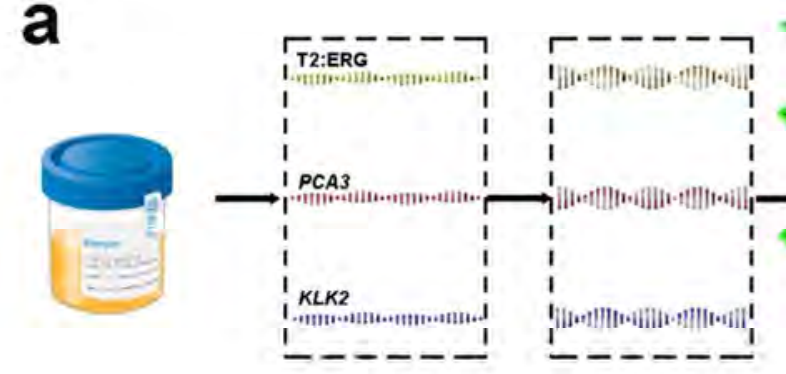

i) Isothermal Amplification of Urinary RNA Targets (T2:ERG, PCA3, KLK2)

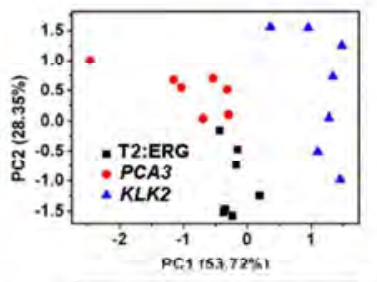

iii) Chemometric Analysis of Unique Target-Specific Signals
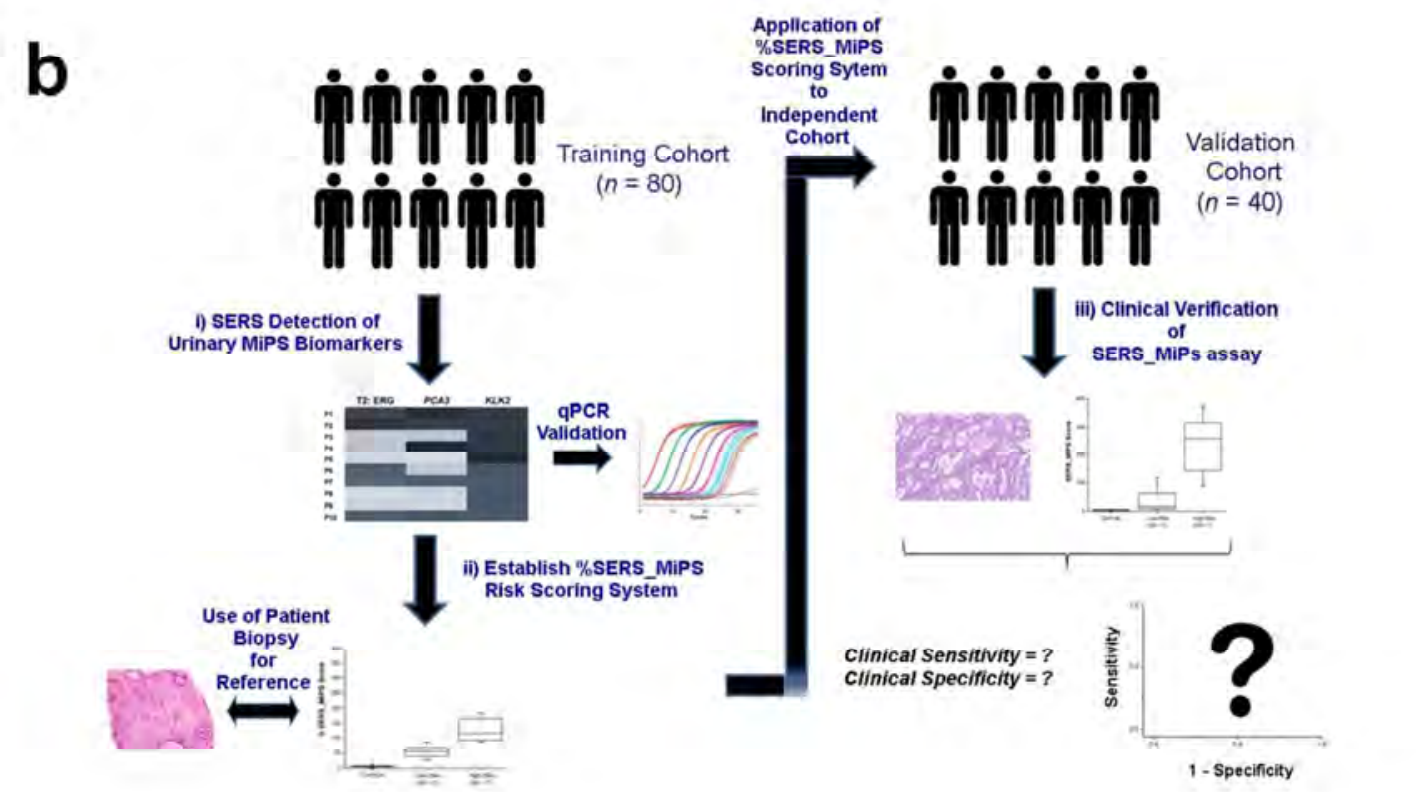

Figure 1. SERS_MiPS detection principle and clinical verification by use of two independent cohorts. a) Extracted patient urinary RNA targets of the clinically-validated MiPS model are isothermally amplified and mixed with cationic SERS substrates for specific target detection. b) A training patient cohort $(n=80)$ is firstly used to establish a \%SERS_MiPS risk scoring system with clinically-relevant cut off limits by relating target SERS signal levels to known patient biopsy outcomes. The \%SERS_MiPS cut off limits are then tested on a validation patient cohort $(n=40)$ to obtain clinical metrics. 

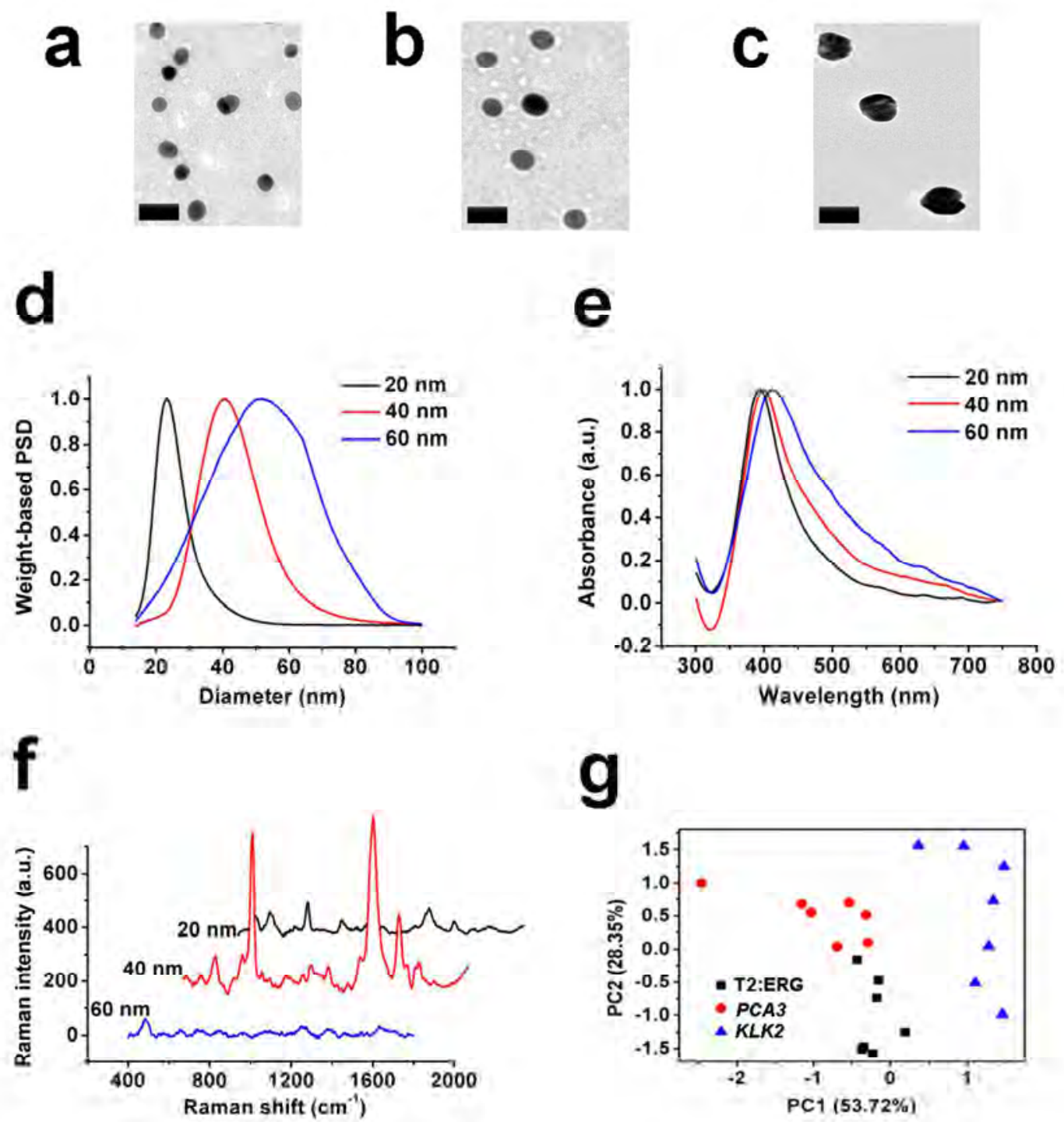

Figure 2. Characterization, optimization and chemometric analysis of SERS substrate. TEM images of synthesized cationic AgNPs of a) $20 \mathrm{~nm}$, b) $40 \mathrm{~nm}$, and $60 \mathrm{~nm}$. Scale bar represents $50 \mathrm{~nm}$. d) Differential centrifugal sedimentation measurements of synthesized cationic AgNP size based on particle weight. e) Surface plasmon resonance bands showing a red-shift with increasing cationic AgNP size. f) SERS measurements of T2:ERG target with different sizes of cationic AgNPs. g) Principle component analysis of SERS spectra from T2:ERG, $P C A 3, K L K 2$ targets. 
a

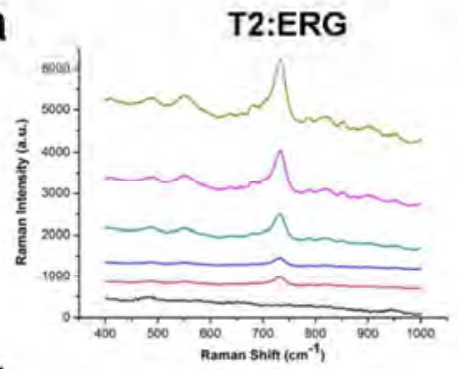

d

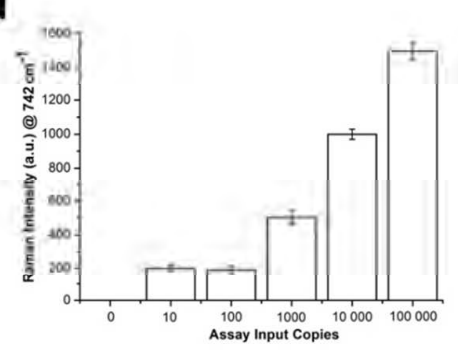

b

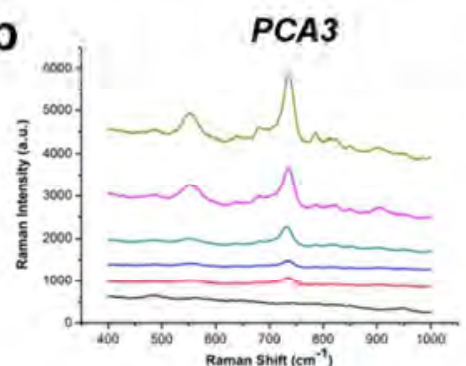

e

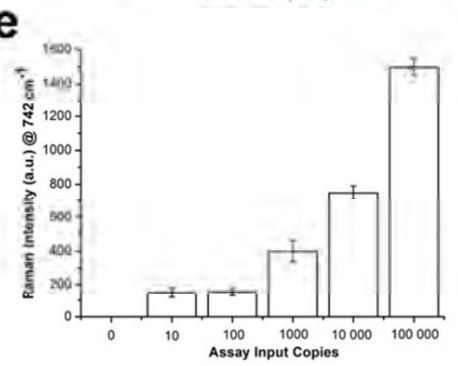

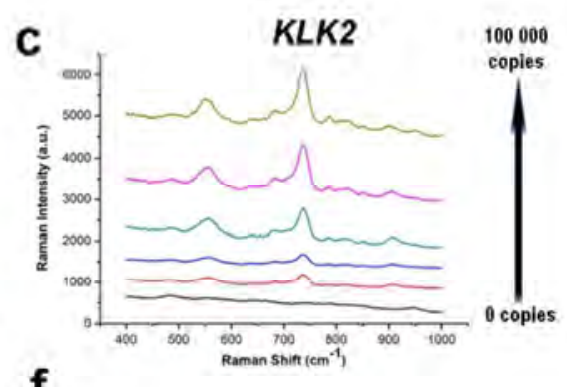
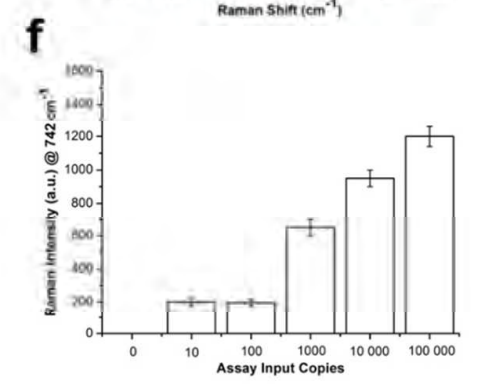

Figure 3. Analytical Sensitivity. SERS spectra of a) T2:ERG, b) $P C A 3$, and c) $K L K 2$ RNA targets at $0-100000$ assay input copies. SERS signal bar plots of d) T2:ERG; e) PCA3; and f) $K L K 2$ at $0-100000$ assay input copies. Error bars represent standard deviations of triplicate independent measurements. 
a

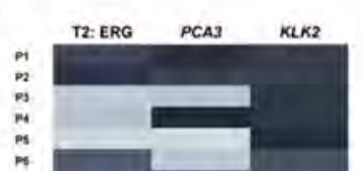

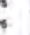

po

${ }_{p 10}^{p}$

P11

$P 11$
$p 12$
$P 11$
$p 16$
$p 16$

pie

pis

P10

pas

${ }_{p m}^{m i}$

pra

par

$\infty$

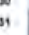

mas

Pas

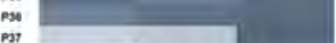

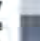

Pat

sat

pes

os

pese

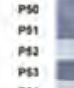

${ }_{m a t}^{\infty}$

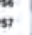

Pas

mo

ค61

mos

Pes

คe?

po

$m 11$
$m 2$
$m m$
$p 74$
$p 74$

Pes

$m$

$m$

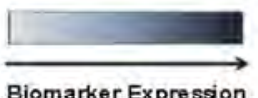

Level

b

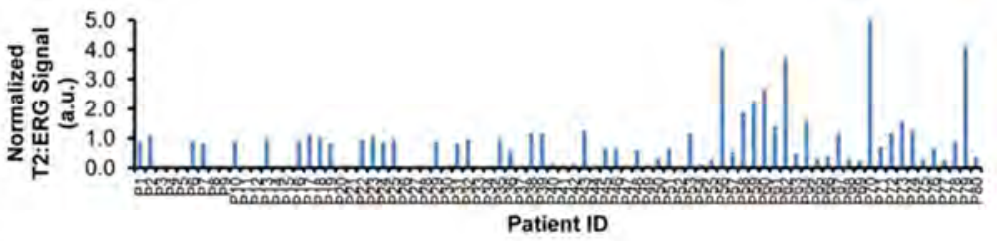

C

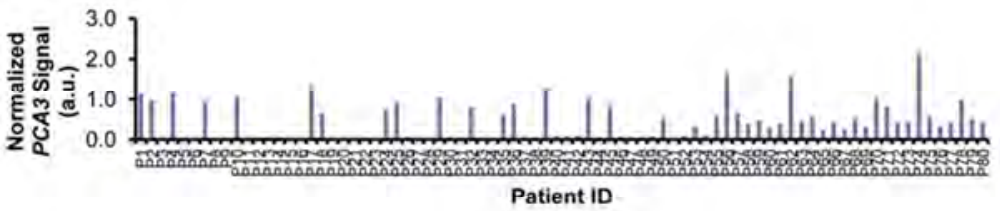

d

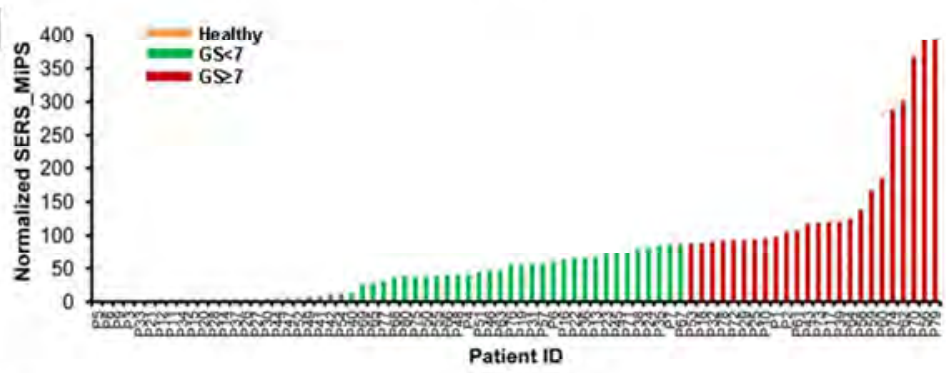

e

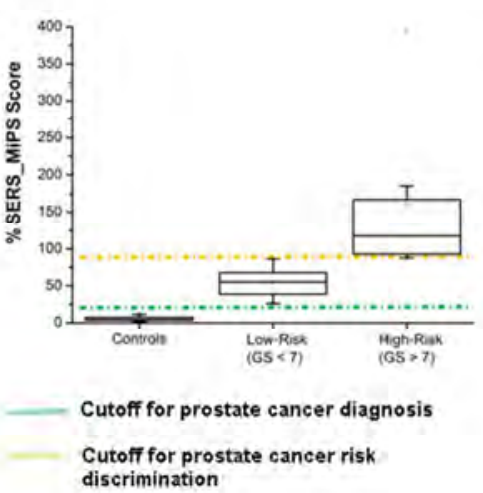

f

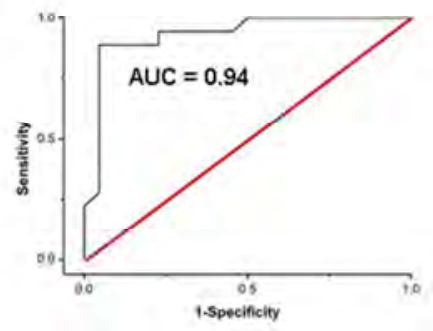

Figure 4. Disease risk scoring in training cohort. a) Heat map representation of T2:ERG, $P C A 3$, and KLK2 SERS signals in urine samples $(n=80)$. Bar plots of b) T2:ERG and c) PCA3 SERS signals after normalization to KLK2 SERS signals. d) Normalized T2:ERG and PCA3 SERS signals are combined to calculate a SERS_MiPS value for each patient. e) An overall \%SERS_MiPS score system is produced by relating SERS_MiPS values to patient biopsy outcomes. f) ROC plot of \%SERS_MiPS to predict high-risk PCa. 

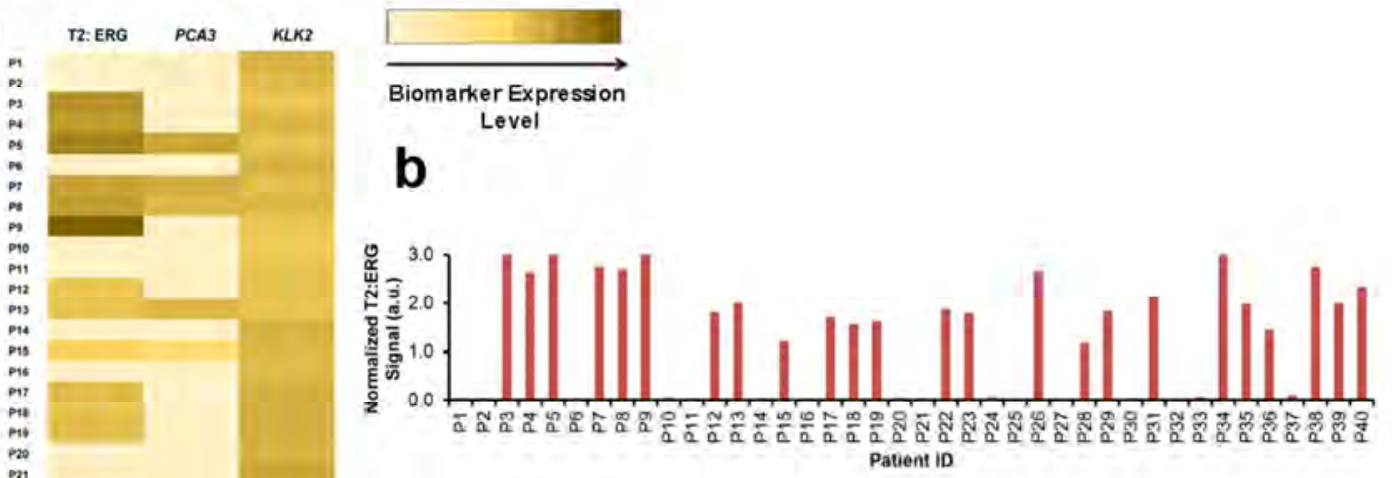

\section{C}
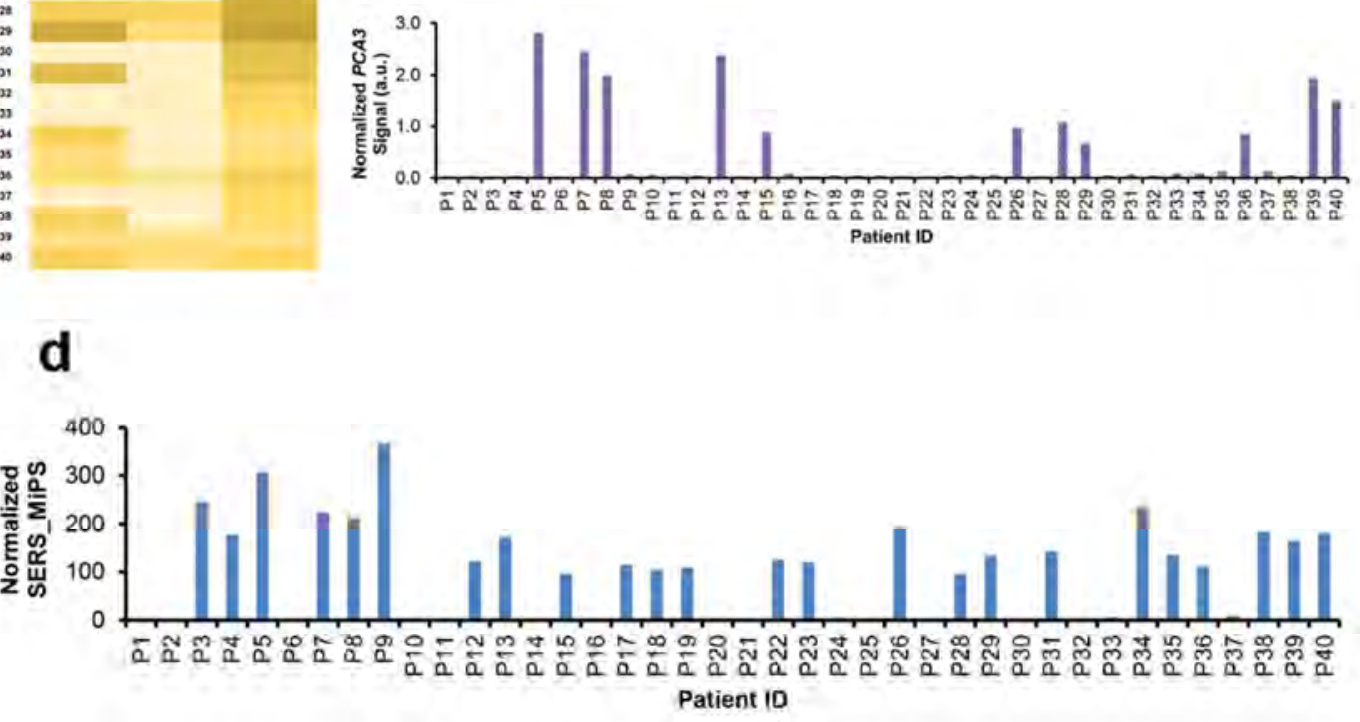

e

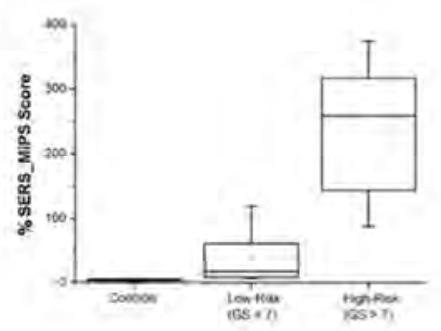

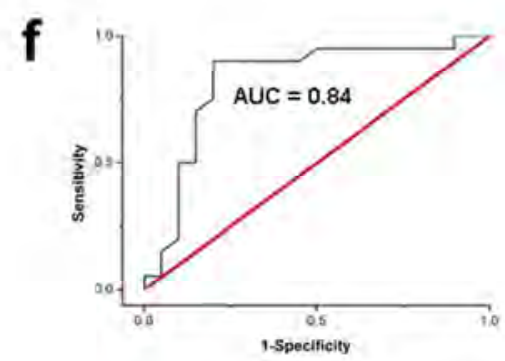

Figure 5. Clinical metric evaluation in validation cohort. a) Heat map representation of T2:ERG, $P C A 3$, and KLK2 SERS signals in urine samples $(n=40)$. Bar plots of b) T2:ERG and c) PCA3 SERS signals after normalization to KLK2 SERS signals. d) Normalized T2:ERG and PCA3 SERS signals are combined to calculate a SERS_MiPS value for each patient. e) An overall \%SERS_MiPS score system is produced by using established cut offs from training cohort. f) ROC plot of \%SERS_MiPS to predict high-risk PCa. 


\section{REFERENCES}

1. Han, M. Y.; Gao, X. H.; Su, J. Z.; Nie, S., Quantum-Dot-Tagged Microbeads for Multiplexed Optical Coding of Biomolecules. Nat. Biotechnol. 2001, 19, 631-635.

2. Cao, Y. W. C.; Jin, R. C.; Mirkin, C. A., Nanoparticles with Raman Spectroscopic Fingerprints for DNA and RNA Detection. Science 2002, 297, 1536-1540.

3. Koo, K. M.; Wee, E. J.; Mainwaring, P. N.; Wang, Y.; Trau, M., Toward Precision Medicine: A Cancer Molecular Subtyping Nano-Strategy for RNA Biomarkers in Tumor and Urine. Small 2016, 12, 6233-6242.

4. Chen, Y. S.; Zhang, Y. X.; Pan, F.; Liu, J.; Wang, K.; Zhang, C. L.; Cheng, S. L.; Lu, L. G.; Zhang, W.; Zhang, Z.; Zhi, X.; Zhang, Q.; Alfranca, G.; de la Fuente, J. M.; Chen, D.; Cui, D. X., Breath Analysis Based on Surface-Enhanced Raman Scattering Sensors Distinguishes Early and Advanced Gastric Cancer Patients from Healthy Persons. ACS Nano 2016, 10, 8169-8179.

5. Chan, W. C. W.; Udugama, B.; Kadhiresan, P.; Kim, J.; Mubareka, S.; Weiss, P. S.; Park, W. J., Patients, Here Comes More Nanotechnology. ACS Nano 2016, 10, 8139-8142.

6. Williams, P. M.; Lively, T. G.; Jessup, J. M.; Conley, B. A., Bridging the Gap: Moving Predictive and Prognostic Assays from Research to Clinical Use. Clin. Cancer Res. 2012, 18, 1531-1539.

7. Kim, J.; Biondi, M. J.; Feld, J. J.; Chan, W. C. W., Clinical Validation of Quantum Dot Barcode Diagnostic Technology. ACS Nano 2016, 10, 4742-4753.

8. van Lierop, D.; Krpetic, Z.; Guerrini, L.; Larmour, I. A.; Dougan, J. A.; Faulds, K.; Graham, D., Positively Charged Silver Nanoparticles and Their Effect on Surface-Enhanced Raman Scattering of Dye-Labelled Oligonucleotides. Chem. Commun. 2012, 48, 8192-8194.

9. Xu, L. J.; Lei, Z. C.; Li, J. X.; Zong, C.; Yang, C. J.; Ren, B., Label-Free SurfaceEnhanced Raman Spectroscopy Detection of DNA with Single-Base Sensitivity. J. Am. Chem. Soc. 2015, 137, 5149-5154.

10. Guerrini, L.; Krpetic, Z.; van Lierop, D.; Alvarez-Puebla, R. A.; Graham, D., Direct Surface-Enhanced Raman Scattering Analysis of DNA Duplexes. Angew. Chem., Int. Ed. 2015, 54, 1144-1148.

11. Morla-Folch, J.; Xie, H. N.; Gisbert-Quilis, P.; Gomez-de Pedro, S.; Pazos-Perez, N.; Alvarez-Puebla, R. A.; Guerrini, L., Ultrasensitive Direct Quantification of Nucleobase Modifications in DNA by Surface-Enhanced Raman Scattering: The Case of Cytosine. Angew. Chem., Int. Ed. 2015, 54, 13650-13654.

12. Koo, K. M.; McNamara, B.; Wee, E. J. H.; Wang, Y.; Trau, M., Rapid and Sensitive Fusion Gene Detection in Prostate Cancer Urinary Specimens by Label-Free SurfaceEnhanced Raman Scattering. J. Biomed. Nanotechnol. 2016, 12, 1798-1805.

13. Morla-Folch, J.; Gisbert-Quilis, P.; Masetti, M.; Garcia-Rico, E.; Alvarez-Puebla, R. A.; Guerrini, L., Conformational SERS Classification of K-Ras Point Mutations for Cancer Diagnostics. Angew. Chem., Int. Edit. 2017, 56, 2381-2385.

14. Morla-Folch, J.; Xie, H. N.; Alvarez-Puebla, R. A.; Guerrini, L., Fast Optical Chemical and Structural Classification of RNA. ACS Nano 2016, 10, 2834-2842.

15. Wang, J.; Koo, K. M.; Wee, E. J. H.; Wang, Y. L.; Trau, M., A Nanoplasmonic Label-Free Surface-Enhanced Raman Scattering Strategy for Non-Invasive Cancer Genetic Subtyping in Patient Samples. Nanoscale 2017, 9, 3496-3503.

16. US Preventive Services Task Force; Grossman, D. C.; Curry, S. J.; Owens, D. K.; Bibbins-Domingo, K.; Caughey, A. B.; Davidson, K. W.; Doubeni, C. A.; Ebell, M.; Epling, J. W. Jr.; Kemper, A. R.; Krist, A. H.; Kubik, M.; Landefeld, C. S.; Mangione, C. M.; Silverstein, M.; Simon, M. A.; Siu, A. L.; Tseng, C. W., Screening for Prostate Cancer: 
US Preventive Services Task Force Recommendation Statement. JAMA 2018, 319, 19011913.

17. Pinsky, P. F.; Prorok, P. C.; Kramer, B. S., Prostate Cancer Screening - A Perspective on the Current State of the Evidence. N. Engl. J. Med. 2017, 376, 1285-1289.

18. Pinsky, P. F.; Prorok, P. C.; Yu, K.; Kramer, B. S.; Black, A.; Gohagan, J. K.; Crawford, E. D.; Grubb, R. L.; Andriole, G. L., Extended Mortality Results for Prostate Cancer Screening in the PLCO Trial with Median Follow-Up of 15 Years. Cancer 2017, 123, 592-599.

19. Carlsson, S. V.; Kattan, M. W., Prostate Cancer: Personalized Risk - Stratified Screening or Abandoning It Altogether? Nat. Rev. Clin. Oncol. 2016, 13, 140-142.

20. Salami, S. S.; Schmidt, F.; Laxman, B.; Regan, M. M.; Rickman, D. S.; Scherr, D.; Bueti, G.; Siddiqui, J.; Tomlins, S. A.; Wei, J. T.; Chinnaiyan, A. M.; Rubin, M. A.; Sanda, M. G., Combining Urinary Detection of TMPRSS2:ERG and PCA3 with Serum PSA to Predict Diagnosis of Prostate Cancer. Urol Oncol.-Semin. Original Invest. 2013, 31, 566-571. 21. Tomlins, S. A.; Day, J. R.; Lonigro, R. J.; Hovelson, D. H.; Siddiqui, J.; Kunju, L. P.; Dunn, R. L.; Meyer, S.; Hodge, P.; Groskopf, J.; Wei, J. T.; Chinnaiyan, A. M., Urine TMPRSS2:ERG Plus PCA3 for Individualized Prostate Cancer Risk Assessment. Eur. Urol. 2015, 70, 45-53.

22. Leyten, G.; Hessels, D.; Jannink, S. A.; Smit, F. P.; de Jong, H.; Cornel, E. B.; de Reijke, T. M.; Vergunst, H.; Kil, P.; Knipscheer, B. C.; van Oort, I. M.; Mulders, P. F. A.; Hulsbergen-van de Kaa, C. A.; Schalken, J. A., Prospective Multicentre Evaluation of PCA3 and TMPRSS2-ERG Gene Fusions as Diagnostic and Prognostic Urinary Biomarkers for Prostate Cancer. Eur. Urol. 2014, 65, 534-542.

23. Cornu, J.-N.; Cancel-Tassin, G.; Egrot, C.; Gaffory, C.; Haab, F.; Cussenot, O., Urine TMPRSS2:ERG Fusion Transcript Integrated with PCA3 Score, Genotyping, and Biological Features Are Correlated to the Results of Prostatic Biopsies in Men at Risk of Prostate Cancer. Prostate 2013, 73, 242-249.

24. Tomlins, S. A.; Aubin, S. M. J.; Siddiqui, J.; Lonigro, R. J.; Sefton-Miller, L.; Miick, S.; Williamsen, S.; Hodge, P.; Meinke, J.; Blase, A.; Penabella, Y.; Day, J. R.; Varambally, R.; Han, B.; Wood, D.; Wang, L.; Sanda, M. G.; Rubin, M. A.; Rhodes, D. R.; Hollenbeck et al., Urine TMPRSS2:ERG Fusion Transcript Stratifies Prostate Cancer Risk in Men with Elevated Serum PSA. Science Transl. Med. 2011, 3, 94ra72.

25. Gisbert-Quilis, P.; Masetti, M.; Morla-Folch, J.; Fitzgerald, J. M.; Pazos-Perez, N.; Garcia-Rico, E.; Giannini, V.; Alvarez-Puebla, R. A.; Guerrini, L., The Structure of Short and Genomic DNA at the Interparticle Junctions of Cationic Nanoparticles. Adv. Mat. Interfaces 2017, 4, 1700724.

26. Torres-Nunez, A.; Faulds, K.; Graham, D.; Alvarez-Puebla, R. A.; Guerrini, L., Silver Colloids as Plasmonic Substrates for Direct Label-Free Surface-Enhanced Raman Scattering Analysis of DNA. Analyst 2016, 141, 5170-5180.

27. Groskopf, J.; Aubin, S. M. J.; Deras, I. L.; Blase, A.; Bodrug, S.; Clark, C.; Brentano, S.; Mathis, J.; Pham, J.; Meyer, T.; Cass, M.; Hodge, P.; Macairan, M. L.; Marks, L. S.; Rittenhouse, H., APTIMA PCA3 Molecular Urine Test: Development of a Method to Aid in the Diagnosis of Prostate Cancer. Clin. Chem. 2006, 52, 1089-1095.

28. Koo, K. M.; Wee, E. J. H.; Wang, Y.; Trau, M., Enabling Miniaturised Personalised Diagnostics: From Lab-on-a-Chip to Lab-in-a-Drop. Lab Chip 2017, 17, 3200-3220.

29. Passing, H.; Bablok, W. A New Biometrical Procedure for Testing the Equality of Measurements from Two Different Analytical Methods. Application of Linear Regression Procedures for Method Comparison Studies in Clinical Chemistry, Part I. J. Clin. Chem. Clin. Biochem. 1983, 21, 709-720. 
30. Zhao, J.; Lui, H.; McLean, D. I.; Zeng, H. Automated Autofluorescence Background Subtraction Algorithm for Biomedical Raman Spectroscopy. Appl. Spectrosc. 2007, 61, $1225-1232$. 


\section{For ToC Only}
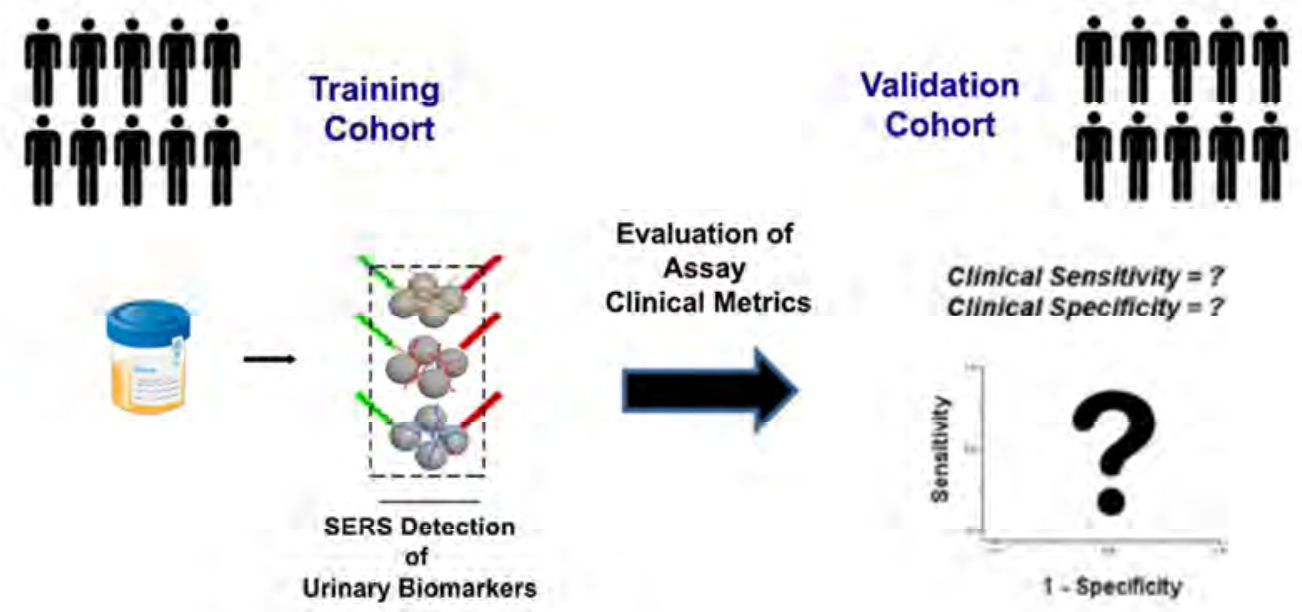